\title{
Development of advanced electron optical systems for novel gyrotrons
}

\author{
V.N. Manuilov ${ }^{1,3}$, G.G. Sominskii ${ }^{2}$ and M.Yu. Glyavin ${ }^{1}$ \\ ${ }^{1}$ Lobachevsky State University, Nizhny Novgorod, Russia, manuilov@rf.unn.ru \\ ${ }^{2}$ Peter the Great St. Petersburg Polytechnic University, St.Petersburg, Russia \\ ${ }^{3}$ Institute of Applied Physics, Nizhny Novgorod, Russia
}

\section{Introduction}

At present the application area of gyrotrons becomes every year wider and covers a lot of new directions of activity such as microwave and CVD technologies, ECRH systems, biology, spectroscopy and diagnostic of various media, hidden objects and defects identification and some others. The number of applications gradually grows and it leads to new, more stringent requirements to the power range, efficiency and frequency stability of gyrotrons. Of course, the mentioned requirements change, to some extent, the traditional scheme of the gyrotron and consequently lead to new versions and trends in the development of corresponding helical electron beam formation systems and types of the gyrotron collectors. Below the main directions in the development of new gyrotron electron optic systems is briefly described.

\section{Results}

The classical scheme of the gyrotron electron-optic system (EOS) includes the adiabatic magnetron injection gun (MIG) and cylindrical or conical collector to dissipate the rest energy of electrons after interaction with RF field in the cavity. The last one may apply the one-stage depressed collector scheme to increase the total efficiency of the gyrotron. The main modern demands to the new generation of gyrotrons are:

1) to reduce velocity spread in HEBs, especially in millimeter and submillimeter wave range;

2) to diminish the influence of reflected from magnetic mirror electrons;

3) to make the EOS less sensitive to the thermal expansion caused by high cathode temperature;

4) to make the device inertia-free, i.e. diminish the time of the switch on/off process;

5) to improve the mode competition problem and due to that reach $1 \mathrm{THz}$ benchmark in $\mathrm{CW}$ operation regime;

6) to increase the total gyrotron efficiency and decrease the temperature load of the collector.

First two problems seem to be solved by turning to the non-adiabatic systems [1] or/and changing the regime of temperature limitation of emission to the space charge limiting regime. Two projects of the non-adiabatic EOS for $28 \mathrm{GHz} / \mathrm{CW}$ technological gyrotron and megawatt power level $170 \mathrm{GHz} / \mathrm{CW}$ are considered (see fig.1, 2). Optimization of the magnetic systems and the electrode shape is performed. The possibility to use the space charge limiting regime is examined.

Next two problems ( 3 and 4) can be solved, in particular, by applying the non-adiabatic systems [1] jointly with field emitters [2] instead of traditional hot cathodes. Such approach is illustrated by example of the gyrotron with an operating frequency of $263 \mathrm{GHz}$, an accelerating voltage of $15 \mathrm{kV}$ and output power some tens watt aimed to use for the spectroscopy applications (fig. 3). Simulation shows that for optimized version of the electron gun the pitch-factor and velocity spread remains close to 1.2 and $30 \%$ respectively in the range of currents $0-50 \mathrm{~mA}$ that is quite admissible to achieve a typical efficiency of a few percent for such gyrotrons.

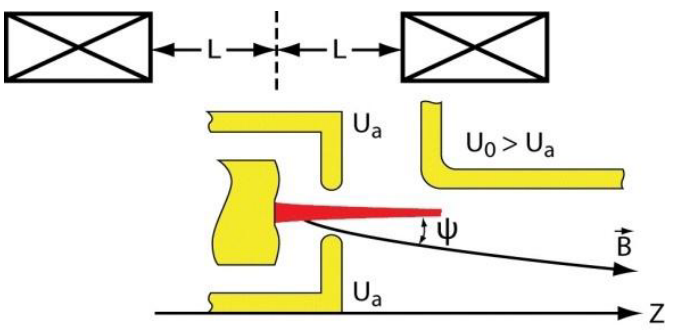

Fig. 1. The principal scheme of the HEB formation region in non-adiabatic EOS. Cathode counterrunning coils to adjust the pitch-factor value are shown

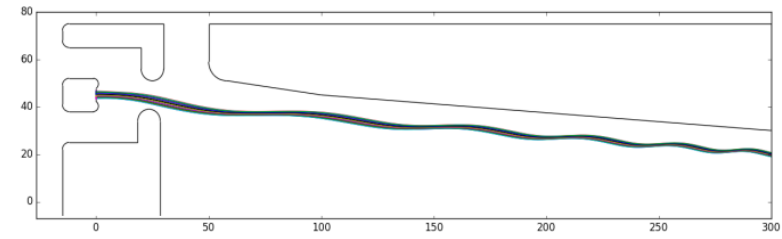

Fig. 2. The configuration of the electrodes in the triode nonadiabatic electron-optical gun for $170 \mathrm{GHz} / 1 \mathrm{MW}$ gyrotron (all dimensions are in $\mathrm{mm}$ )

The only way to reach $1 \mathrm{THz} \mathrm{CW}$ operation and at the same time to keep reasonable cost of the magnetic system and the gyrotron itself is to use high harmonic operation. One the effective ways to solve mode competition problem in such case is to use multi-beam gyrotrons [3] (item 5 in the list presented above). In the report the possible schemes of the multi-beam EOS are presented. The example of the two-beam adiabatic formation system with both generating beams for $790 \mathrm{GHz} / \mathrm{CW}$ second harmonic gyrotron is described and optimized (fig. 4). For a more flexible control of the parameters of the partial HEBs a triode MIG with an anode voltage of $U_{\mathrm{a}} \approx 10 \mathrm{kV}$ has been envisaged. It has been assumed that both partial beams are laminar (with an incidence angle of the magnetic field on the emitting surface $\varphi \approx 25^{\circ}$ ) in order to reduce the influence of the space-charge field and have equal pitch factors $g_{1,2} \approx 1.3$. The currents of the two beams relate as 1:2. Aiming a sufficient longevity of the cathode the current density extracted from the emitter is limited to $2 \mathrm{~A} / \mathrm{cm}^{2}$.

The total gyrotron based installation efficiency is extremely important for industrial $\mathrm{CW}$ gyrotrons. The total efficiency depends on the interaction one, efficiency of 
energy recovery and power consumption aimed to produce the operating magnetic field (item 6). In the report the idea to develop the gyrotron EOS with hot magnet surrounding by ferromagnetic screens is described (fig. 5). For fixed value of the operating magnetic field it allowed to decrease the power of the main coil in two times approximately. Optimization of the coils and screens geometry allowed to provide the inhomogeneity of the axial magnetic field in the cavity less than 1\%. Rapid drop of the magnetic field outside the screens breaks the adiabatic conditions for magnetic field in the formation region. It makes difficult to use well known and reliable MIG for HEB generation. To keep the adiabaticity of the field the additional small power cathode coil is included into the scheme of EOS (fig. 5). Optimized MIG geometry provides high pitch-factor $\mathrm{g}>1.6$ and at the same time modest velocity spread in wide range of the operating regimes.

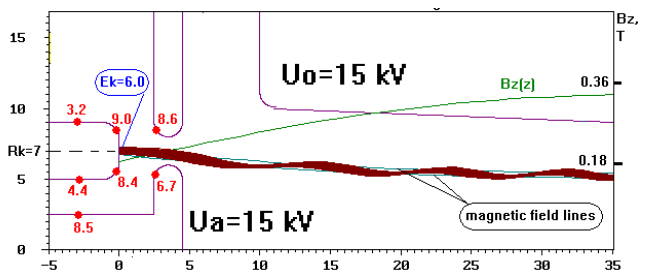

a)

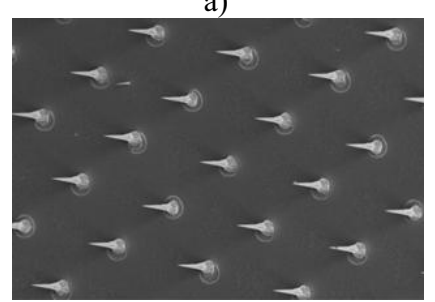

b)

Fig. 3. Scheme of the primary formation of the beam in a nonadiabatic EOS (a). Distributions of the axial magnetic field B(z) (right axis, data are in $\mathrm{T}$ ) is presented. Dots and corresponding numbers show the electric field value $(\mathrm{kV} / \mathrm{mm})$ at the most critical points. Electric field Ek at the emitter center is also specified. All dimensions are in $\mathrm{mm}$. The multi-pin stricture of the cathode (b) is shown

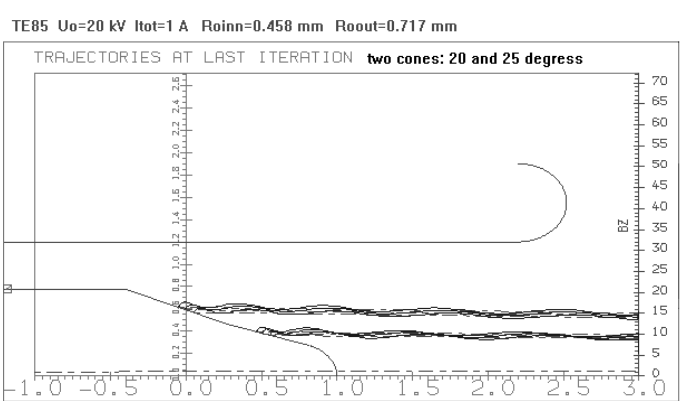

Fig. 4. The electrode profile for double-beam MIG with two generating beams
Rapid decrease of the magnetic field intensity outside the ferromagnetic screens allows also apply the new scheme of the collector with inertial expansion of the HEB and so to decrease the power density on the collector. The results of numerical simulation of such scheme are briefly discussed.

Conventional powerful gyrotron scheme includes also the one-stage CPD collector. But the corresponding gyrotron efficiency usually limited by the value $50-55 \%$. Further increase of the CPD collector efficiency can be reached by turning to two-stage scheme. Here the main and very difficult problem is the space separation of the electrons with different energies. Some ways to solve it (collectors with crossed electric and magnetic fields, with reverse of the magnetic field) are presented in the rest part of the report.

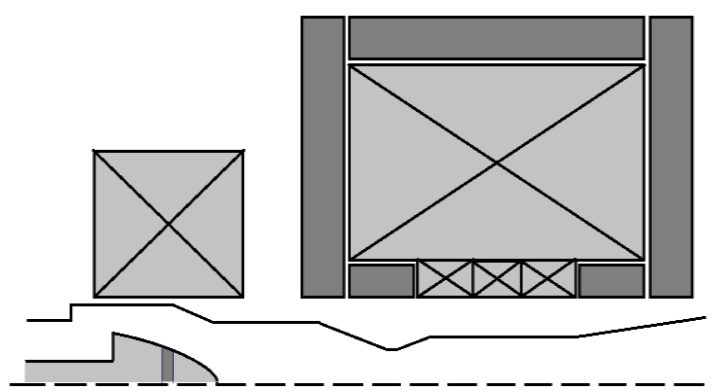

Fig. 5. Scheme of the electron optic system with ferromagnetic screens and magnetron-injection gun

\section{Conclusion}

Some new helical electron beam formation systems as well as the new concepts of collectors for gyrotrons are described and examined. It is shown that they open new promising possibilities in the development of new generation of gyrotrons with improved efficiency and increased frequency and stability.

\section{Acknowledgements}

The work was supported by the Russian Science Foundation, project No. 14-12-00887.

\section{References}

1. A.L. Goldenberg, V.N. Manuilov and M.Yu. Glyavin. Electron-Optical System of a High-Power Gyrotron with Nonadiabatic Electron Gun // Radiophysics and Quantum Electronics, vol.48, No 6, 2005, pp. 461-465

2. G.G. Sominskii, T.A. Tumareva, E.P. Taradaev, M.V. Mishin, A.N. Stepanova. Multitip semiconductor field emitters with new-type bilayer protecting coatings. // Tech. Phys., 60(1), 133-136 (2015).

3. V.E. Zapevalov, Sh.E. Tsimring. Multibeam gyrotrons. // Radiophys. Quantum Electron. 33, 11, 954-960 (1990). 